\title{
Modelagem estatística para a estimativa mensal de horas e unidades de frio em regiões de planalto do Rio Grande do Sul e Santa Catarina
}

\author{
Rosandro Boligon Minuzzi ${ }^{1}$ \\ $10.1590 / 0034-737 X 201865060002$
}

\begin{abstract}
RESUMO
O objetivo deste estudo foi obter e avaliar modelos para a estimativa mensal de horas- unidades de frio, com base em dados de temperatura do ar, nas regiões de planalto do Rio Grande do Sul e de Santa Catarina. Dados horários de temperatura do ar foram usados, no período de maio a agosto de 2012 a 2017, de sete estações meteorológicas. Para cada mês, foram calculadas as horas-unidades de frio pelos métodos de Utah e da soma de temperaturas horárias iguais ou inferiores a $7,2^{\circ} \mathrm{C}$, que foram correlacionados com a temperatura mínima e média mensal, com o uso da análise de regressão. Seis métodos estatísticos foram usados para avaliar a estimativa mensal de horas-unidades de frio, obtidas pelos modelos previamente escolhidos. A temperatura mínima e a média mensal explicaram entre 65 e $96 \%$ das horas de frio, pelo limiar de temperatura $\leq 7,2{ }^{\circ} \mathrm{C}$, e as unidades de frio pelo método de Utah. A temperatura mínima e a média mensal do ar são recomendadas para a estimativa das horas de frio e das unidades de frio, respectivamente. A variabilidade climática do inverno na região em estudo resulta em desempenhos distintos dos modelos de estimativas de horas- unidades de frio.
\end{abstract}

Palavras-chave: superação da dormência; fruticultura, método de Utah.

\section{ABSTRACT \\ Statistical modeling for the monthly estimate of chilling hours and units in Plateau regions of Rio Grande do Sul and Santa Catarina, Brazil}

The objective of this study was to obtain and evaluate models for the monthly estimation of chilling hours/units based on air temperature data in the Plateau regions of Rio Grande do Sul and Santa Catarina. Data of air temperature were used from May to August 2012 to 2017 of seven meteorological stations. For each month, the chilling hours/unit were calculated by the Utah method and the sum of temperatures lower or equal to $7.2{ }^{\circ} \mathrm{C}$ that were correlated with the minimum and average monthly temperature by the regression analysis. Six statistical methods were used to evaluate the monthly estimate of chilling hours/units obtained by previously obtained models. The minimum temperature and the monthly average explained between 65 and $96 \%$ of the chilling hours by the temperature threshold d" $7.2{ }^{\circ} \mathrm{C}$ and the chilling units by the Utah method. The minimum temperature and the monthly air average are recommended for the estimation of chilling hours and chilling units, respectively. The climatic variability of the winter in the study region results in different performances of the models of estimates of chilling hours/units.

Keywords: breaking dormancy; fruticulture; Utah method.

\footnotetext{
Submetido em 02//03/2018 e aprovado em 13/11/2018.

${ }^{1}$ Universidade Federal de Santa Catarina, Departamento de Engenharia Rural, Florianópolis, Santa Catarina, Brasil. rbminuzzi@hotmail.com Catarina, Brasil.rbminuzzi@hotmail.com

*Autor para correspondência: rbminuzzi@hotmail.com
} 


\section{INTRODUÇÃO}

A temperatura do ar influencia diretamente o crescimento e o desenvolvimento vegetal. Os processos internos de transporte e o efeito na velocidade das reações químicas somente ocorrem dentro de limites térmicos nos quais a tolerância é variável (Pereira et al., 2002).

Espécies frutíferas de clima temperado de folhas caducas têm período de repouso invernal, em que não ocorre crescimento vegetativo. Esse processo é condicionado pelas condições climáticas atuantes sobre os reguladores de crescimento. Após a fase de dormência, um novo ciclo vegetativo deve- se iniciar, desde que a planta tenha acumulado uma determinada quantidade de frio. A insuficiência de frio hibernal deixa as plantas sujeitas a algumas anomalias, como especificam Fioravanço \& Santos (2013) para a macieira. A cultura pode permanecer em dormência ou apresentar brotação e floração reduzidas e, ou, desuniformes. Como consequência, as plantas apresentam comprometimentos na distribuição dos ramos, na formação de superfície foliar adequada e no potencial produtivo da safra, com formação de frutos pequenos e achatados.

Na relação da aptidão climática com a produtividade agrícola dependente do acúmulo de horas-unidades de frio, o planalto gaúcho e catarinense tem destaque no cultivo de maçã. De 2014 a 2017, o Brasil produziu em média 1.248.366 toneladas de maçã, sendo Santa Catarina e Rio Grande do Sul responsáveis por $96,2 \%$ da produção nacional (IBGE, 2018).

A modelagem de previsão do início do ciclo vegetativo anual, em frutíferas caducifólias, por meio da quantificação do frio, é de extrema importância para a tomada de decisão quanto à necessidade, ou não, de aplicação de produtos químicos para a superação da dormência. Além disso, auxilia na administração de doses adequadas às necessidades de uma determinada safra (Fioravanço \& Santos, 2013). Botelho et al. (2006) usaram diferentes modelos, como o de Utah, para indicar uma aptidão climática mais precisa para cultivares de frutíferas de clima temperado, como a maçã, pêssego, ameixa e kiwi, em algumas regiões do Paraná.

Baseando-se em modelos elaborados em países do Hemisfério Norte, adota-se, comumente, o total de horas de frio inferiores a $7,2^{\circ} \mathrm{C}$ como referência para indicação de uma região como apta ou inapta à implantação de uma frutífera caducifólia. Mas Nachtigall et al. (2009) acrescentam que, para regiões de clima subtropical, como no sul do Brasil, outros indicadores, como o de Utah, podem ser tão ou mais eficientes que as horas de frio, já que temperaturas acima de $10{ }^{\circ} \mathrm{C}$ a $12{ }^{\circ} \mathrm{C}$ também têm efeito favorável na superação da dormência, assim como, temperaturas acima de $21{ }^{\circ} \mathrm{C}$, durante o inverno, anulam o efeito do frio previamente acumulado.
No entanto, a modelagem para quantificação de frio exige registros horários de temperatura e o tomador de decisão nem sempre dispõe de termômetros registradores, que oferecem este tipo de informação, ou, para pesquisa em âmbito climático, de uma longa série histórica de dados. Além disso, por causa da oscilação climática, as temperaturas no outono-inverno no sul do Brasil frequentemente são insuficientes para satisfazer às necessidades fisiológicas da dormência.

Diante destas questões, o objetivo deste estudo foi obter e avaliar modelos para a estimativa mensal de horas-unidades de frio, com base em dados de temperatura do ar nas regiões de planalto do Rio Grande do Sul e de Santa Catarina.

\section{MATERIAL E MÉTODOS}

Dados horários de temperatura do ar foram utilizados, no período de maio a agosto de 2012 a 2017, provenientes de algumas estações meteorológicas pertencentes ao Instituto Nacional de Meteorologia (INMET), localizadas nas regiões de planalto do Rio Grande do Sul (RS) e de Santa Catarina (SC), conforme apresentadas na Figura 1. Demais informações acerca das referidas estações, como a altitude, as médias climatológicas das temperaturas médias e mínimas, do período de maio a agosto, e o número de meses usados no estudo, constam na Tabela 1.

Para cada mês útil do quadrimestre maio-agosto, foram calculadas as unidades de frio e as horas de frio, utilizando-se o método de Utah e o que contabiliza o número de horas de temperaturas iguais ou inferiores a $7,2^{\circ} \mathrm{C}$, respectivamente, além da média mensal das temperaturas média e mínima do ar, que foram utilizadas como variáveis preditoras. O método de Utah considera que uma dada temperatura, por uma hora, equivale a uma determinada quantidade de unidades de frio (Tabela 2). Os modelos preditivos para cada um dos municípios foram obtidos com a Análise de Regressão, sendo utilizado como critério para definição da equação de estimativa, aquela com maior coeficiente de determinação, desde que, significativo a $5 \%$ de probabilidade. O software Statistica v. 13 foi utilizado para realizar as análises estatísticas.

Dos municípios com pelo menos 18 meses úteis para o estudo (Tabela 1), entre 33 e $44 \%$ dos meses foram utilizados apenas para avaliar o desempenho dos modelos obtidos.

A avaliação da estimativa mensal de unidades e horas de frio pelos referidos métodos foi realizada por meio do erro padrão da estimativa (EPE), do erro médio (EM), dos coeficientes de correlação (r) e de determinação $\left(R^{2}\right)$ da regressão linear, do índice de concordância (d) e do índice de confiança (c). 
O Índice de Concordância (d) (Willmott, 1981) descrito na Equação 1, varia de 0 a 1 e representa o quanto as unidades-horas de frio, estimadas pelos modelos encontrados ajustam-se aos valores calculados, sendo que valores próximos de 1 indicam uma concordância perfeita.

$\mathrm{d}=1-\left[\frac{\sum_{\mathrm{i}=1}^{\mathrm{N}}\left(\mathrm{Y}_{\mathrm{i}}-\mathrm{X}_{\mathrm{i}}\right)^{2}}{\sum_{\mathrm{i}=1}^{\mathrm{N}}\left(\left|\mathrm{Y}_{\mathrm{i}}-\overline{\mathrm{X}}\right|+\left|\mathrm{X}_{\mathrm{i}}-\overline{\mathrm{X}}\right|\right)^{2}}\right.$

em que $\mathrm{Xi}=$ são os valores de unidades-horas de frio, calculados por Utah e temperaturas $=7,2^{\circ} \mathrm{C}$; é a média dos valores de unidades-horas de frio, calculados por Utah e temperaturas $=7,2{ }^{\circ} \mathrm{C}$; $\mathrm{Yi}=$ são os valores de unidadeshoras de frio, estimados pelos modelos; $\mathrm{N}=$ é o número de meses úteis.

Correlativamente, para a análise da confiabilidade de horas-unidades de frio estimada pelos modelos, considerou-se o Índice de Confiança (c), proposto por Camargo
\& Sentelhas (1997), conforme Equação 2. O critério adotado para interpretar os valores de c, consta da Tabela 3.

$\mathrm{c}=\mathrm{r} \cdot \mathrm{d}$

O cálculo para o erro padrão da estimativa (EPE) foi obtido, utilizando-se a Equação 3:

$\mathrm{EPE}=\sqrt{\left(\frac{\sum_{\mathrm{i}=1}^{\mathrm{N}}\left(\mathrm{Y}_{\mathrm{i}}-\mathrm{X}_{\mathrm{i}}\right)^{2}}{\mathrm{~N}-1}\right)}$

Já o Erro Médio (EM), representa a diferença média de unidades-horas de frio estimada pelos modelos com os valores calculados por Utah e temperatura $=7,2^{\circ} \mathrm{C}$. Assim, o EM indica a possível tendência de as unidadeshoras de frio, estimada pelos modelos a superestimar $(E M>0)$ ou a subestimar $(E M<0)$ os valores calculados, e é calculado conforme a Equação 4:

$\mathrm{EM}=\frac{1}{\mathrm{~N}} \sum_{\mathrm{i}=1}^{\mathrm{N}}\left(\mathrm{Y}_{\mathrm{i}}-\mathrm{X}_{\mathrm{i}}\right)$

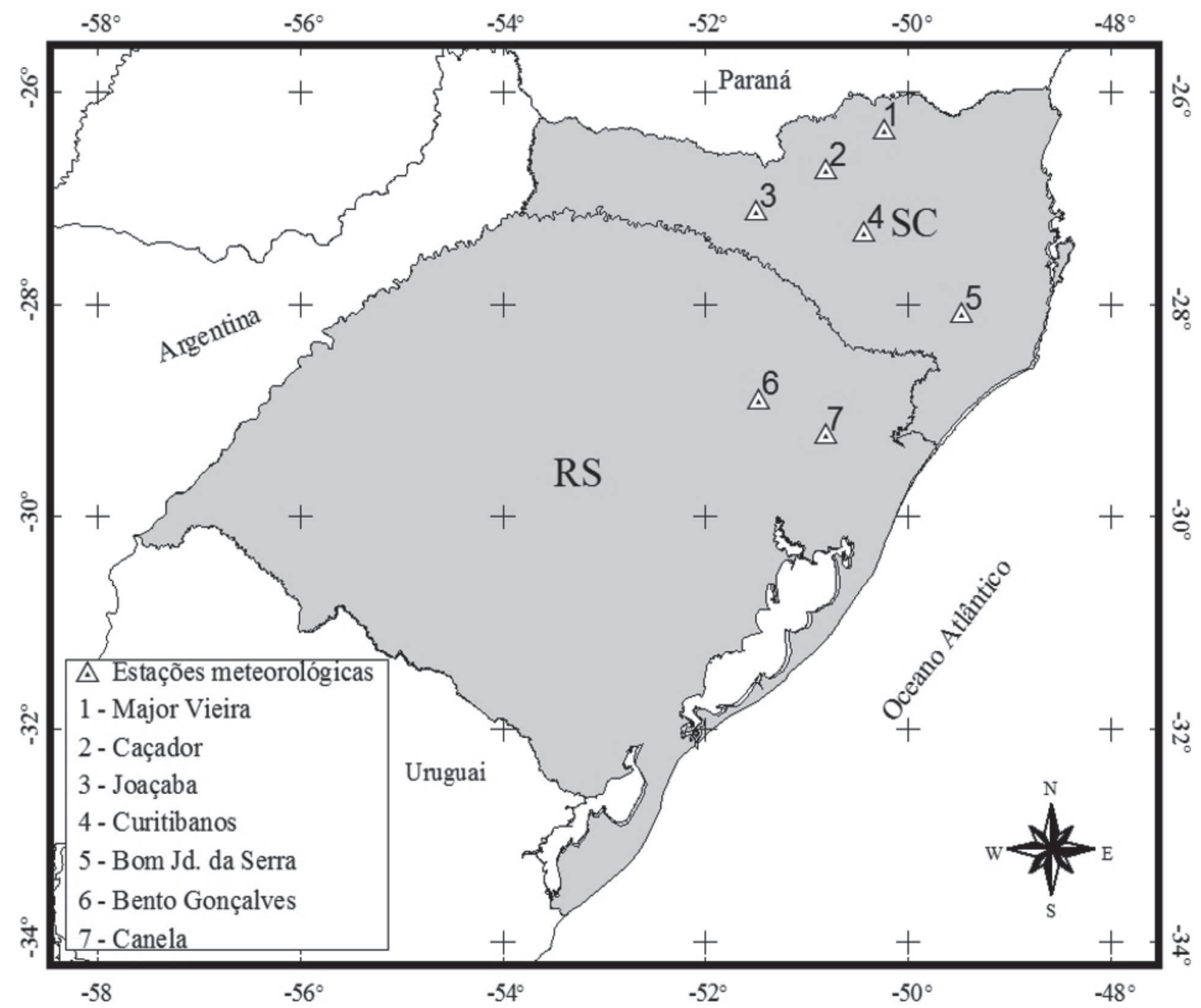

Figura 1: Localização geográfica dos municípios com estações meteorológicas usadas neste estudo, em Santa Catarina (SC) e no Rio Grande do Sul (RS). 
Tabela 1: Altitude e médias climatológicas das temperaturas média e mínima do ar do período de maio a agosto e o número de meses usados no quadrimestre, de 2012 a 2017

\begin{tabular}{lcccc}
\hline Município (Estado) & $\begin{array}{c}\text { Altitude } \\
(\mathbf{m e t r o s})\end{array}$ & $\begin{array}{c}\text { Temperaturamédia } \\
\left({ }^{\circ} \mathbf{C}\right)\end{array}$ & $\begin{array}{c}\text { Temperaturamínima } \\
\left({ }^{\circ} \mathbf{C}\right)\end{array}$ & $\mathbf{N}^{\mathbf{o}}$ meses úteis \\
\hline Bento Gonçalves (RS) & 623 & 13,9 & 10,2 & 18 \\
Bom Jardim da Serra (SC) & 1822 & 11,9 & 7,2 & 20 \\
Caçador (SC) & 944 & 13,2 & 8,4 & 20 \\
Canela (RS) & 831 & 12,2 & 8,7 & 16 \\
Curitibanos (SC) & 982 & 13,9 & 8,8 & 24 \\
Joaçaba (SC) & 768 & 14,5 & 10,2 & 16 \\
Major Vieira (SC) & 800 & 12,5 & 8,1 & 24 \\
\hline
\end{tabular}

\section{RESULTADOS E DISCUSSÃO}

A Tabela 4 apresenta as equações de regressão estatisticamente significativas a 5\%, com os maiores coeficientes de determinação $\left(R^{2}\right)$ entre o acúmulo mensal (maio a agosto) de horas-unidades de frio com a temperatura média e a mínima mensal para os sete municípios usados no estudo. Salvo algumas exceções observadas em Joaçaba, Caçador e Canela, a temperatura mínima e a média mensal do ar conseguem explicar entre 65 e $96 \%$ das horas-unidades de frio.

Numa análise mais específica, por método de quantificação de frio, o método das horas de frio obtidas com o limiar de $7,2^{\circ} \mathrm{C}$ apresentou as melhores correlações com a temperatura mínima $(\mathrm{r}=0,84$ a 0,93$)$, seguido pelo método de Utah, com a temperatura média mensal. Com exceção de Bom Jardim da Serra $(\mathrm{r}=0,81)$ os demais municípios apresentaram correlações de 0,87 a 0,98 . Para quantificar o acúmulo mensal de horas de frio, usando-se o limiar de $7,2{ }^{\circ} \mathrm{C}$ e as unidades de frio por Utah, recomenda-se utilizar a temperatura mínima e a média mensal como variáveis preditoras, respectivamente. A explicação pode estar na climatologia de temperatura do ar para a época do ano na região em estudo (Tabela 1), associada com a forma como ambos os métodos quantificam o frio. Enquanto o método das horas de frio com limiar de $7,2^{\circ} \mathrm{C}$ trabalha com temperaturas mais baixas, o método de Utah é mais abrangente em contabilizar tanto temperaturas mais baixas quanto mais altas, que seria mais cabível para as temperaturas médias da região sul do Brasil. Seguindo este raciocínio, Rea \& Eccel (2006) testaram vários modelos para prever data de florescimento da maçã no norte da Itália. Como, em muitas situações, a precisão foi baixa, os autores desenvolveram uma versão modificada do modelo de Utah, denominada de 'Utah progressivo', no qual o modelo fenológico mostrou-se eficiente mesmo em locais com diferentes microclimas que resultem em datas de florescimento diferentes.

$\mathrm{Na}$ maioria das situações, os modelos obtidos foram oriundos de regressão linear simples e, ou polinomial de segunda ordem. Bento Gonçalves foi o
Tabela 2: Unidades de frio (UF) pelo método de Utah, em função da temperatura do ar horária

\begin{tabular}{lc}
\hline Temperatura horária do ar $\left({ }^{\circ} \mathbf{C}\right)$ & Unidades de frio (UF) \\
\hline$<1,4$ & 0,0 \\
1,5 a 2,4 & 0,5 \\
2,5 a 9,1 & 1,0 \\
9,2 a 12,4 & 0,5 \\
12,5 a 15,9 & 0,0 \\
16,0 a 18,0 & $-0,5$ \\
$>18,0$ & $-1,0$ \\
\hline
\end{tabular}

Tabela 3: Critério de interpretação do índice de confiança (Camargo \& Sentelhas, 1997)

\begin{tabular}{lc}
\hline Índice de confiança $(\mathbf{c})$ & Desempenho \\
\hline$>0,86$ & Ótimo \\
0,76 a 0,85 & Muito Bom \\
0,66 a 0,75 & Bom \\
0,61 a 0,65 & Mediano \\
0,51 a 0,60 & Sofrível \\
0,41 a 0,50 & Mau \\
$\leq 0,40$ & Péssimo \\
\hline
\end{tabular}

único município em que foi necessário recorrer a uma regressão polinomial de terceira ordem para ajustar o modelo de maior correlação.

Linvill (1990) foi mais detalhado em seu estudo, que visou a estimar horas-unidades de frio diárias. O pesquisador encontrou um modelo seno-logarítimo, que necessita da temperatura mínima, da máxima ou da média diárias, que foram bem próximas das determinadas com os registros horários de temperaturas.

Diante dos resultados satisfatórios na obtenção dos modelos, a escolha do método para quantificação do frio depende de vários fatores, como local, cultura, variedade e sua idade.

A favor da utilização do método de Utah constam alguns estudos como o de Felippeto (2008). Após encontrar as maiores correlações entre a variabilidade dos acúmulos de frio, obtidos pelos modelos de Utah e da 
Carolina do Norte, com o número de dias até o início da brotação do cultivar Cabernet Sauvignon, o autor recomendou esses modelos para futuras pesquisas para melhor compreensão da influência térmica sobre a fenologia de outros cultivares de videira de brotação tardia. Erez (2000) ressalta que a temperatura de $10{ }^{\circ} \mathrm{C}$ apresenta metade da eficiência na quebra de dormência de gemas de pessegueiros, quando comparada com a temperatura de $6{ }^{\circ} \mathrm{C}$, e que a temperatura de $21^{\circ} \mathrm{C}$, alternada com baixas temperaturas, nulifica o efeito do frio já acumulado.

Estudo realizado por Pola et al. (1994) para as condições climáticas de Caçador, relativo à brotação da macieira cv. Gala, concluiu que o modelo de horas de frio com limiar de $7,2{ }^{\circ} \mathrm{C}$ apresentou baixa correlação com a data de início da brotação, enquanto os modelos de Carolina do Norte e Carolina do Norte Modificado, que utilizam metodologia semelhante ao de Utah, apresentaram as maiores correlações. Apesar dos objetivos diferentes, neste estudo, o modelo com melhor correlação $(r=0,96)$ para Caçador foi com as unidades de frio obtidas pelo método de Utah (Tabela 4).

As Tabelas 5 e 6 apresentam a avaliação das estimativas de horas-unidades de frio obtidas pelos modelos com maior coeficiente de determinação, usando-se em torno de 33 a $44 \%$ dos meses disponíveis neste estudo. No geral, isto equivale aos meses de maio a agosto de 2016 e 2017.

Tabela 4: Resultado das análises de regressão entre horas de frio $\left(\mathrm{HF}_{7,2}\right)$ e unidades de frio $\left(\mathrm{UF}_{\mathrm{Utah}}\right)$ com as temperaturas média $\left(\mathrm{t}_{\mathrm{md}}\right)$ e mínima $\left(\mathrm{t}_{\min }\right)$ mensal, para os municípios deste estudo

\begin{tabular}{|c|c|c|c|}
\hline & $\mathbf{r}$ & $\mathbf{R}^{2}$ & p-valor \\
\hline \multicolumn{4}{|l|}{ Curitibanos } \\
\hline $\mathrm{HF}_{7,2}=309,6-25,59 \cdot \mathrm{t}_{\text {min }}$ & 0,84 & 0,71 & 0,0000 \\
\hline $\mathrm{HF}_{7,2}=461,1-29,85 \cdot \mathrm{t}_{\mathrm{md}}$ & 0,81 & 0,66 & 0,0002 \\
\hline $\mathrm{UF}_{\mathrm{Utah}}=-569,3+199 \cdot \mathrm{t}_{\min }-13,79 \cdot \mathrm{t}_{\min }{ }^{2}$ & 0,90 & 0,81 & 0,0000 \\
\hline $\mathrm{UF}_{\mathrm{Utah}}=1112-79,76 . \mathrm{t}_{\mathrm{md}}$ & 0,98 & 0,96 & 0,0000 \\
\hline \multicolumn{4}{|l|}{ Bom Jardim da Serra } \\
\hline $\mathrm{HF}_{7,2}=593,3-57,04 \cdot \mathrm{t}_{\min }$ & 0,96 & 0,93 & 0,0000 \\
\hline $\mathrm{HF}_{7,2}=745,8-57,3 \cdot \mathrm{t}_{\mathrm{md}}$ & 0,93 & 0,86 & 0,0000 \\
\hline $\mathrm{UF}_{\mathrm{Utah}}=-522,4+325,1 \cdot \mathrm{t}_{\min }-26,76 \cdot \mathrm{t}_{\min }{ }^{2}$ & 0,81 & 0,65 & 0,0008 \\
\hline $\mathrm{UF}_{\mathrm{Utah}}=-1136+372,6 \cdot \mathrm{t}_{\mathrm{md}}-21,81 \cdot \mathrm{t}_{\mathrm{md}}^{2}$ & 0,81 & 0,66 & 0,0007 \\
\hline \multicolumn{4}{|l|}{ Major Vieira } \\
\hline $\mathrm{HF}_{7,2}=567,9-90,9 \cdot \mathrm{t}_{\min }+3,748 \cdot \mathrm{t}_{\min }{ }^{2}$ & 0,93 & 0,86 & 0,0001 \\
\hline $\mathrm{HF}_{7,2}=1529-189,1 \cdot \mathrm{t}_{\mathrm{md}}+5,889 \cdot \mathrm{t}_{\mathrm{md}}^{2}$ & 0,90 & 0,81 & 0,0000 \\
\hline $\mathrm{UF}_{\mathrm{Utah}}=1,556+61,89 \cdot \mathrm{t}_{\min }-6,321 \cdot \mathrm{t}_{\min }{ }^{2}$ & 0,81 & 0,66 & 0,0074 \\
\hline $\mathrm{UF}_{\mathrm{Utah}}=-376,5+138,9 \cdot \mathrm{t}_{\mathrm{md}}-7,968 \cdot \mathrm{t}_{\mathrm{md}}{ }^{2}$ & 0,97 & 0,94 & 0,0000 \\
\hline \multicolumn{4}{|l|}{ Joaçaba } \\
\hline $\mathrm{HF}_{7,2}=310,8-24,48 \cdot \mathrm{t}_{\min }$ & 0,92 & 0,85 & 0,0000 \\
\hline $\mathrm{HF}_{7,2}=729,2-76,04 \cdot \mathrm{t}_{\mathrm{md}}+2,038 . \mathrm{t}_{\mathrm{md}}^{2}$ & 0,66 & 0,43 & 0,0192 \\
\hline $\mathrm{UF}_{\mathrm{Utah}}=527,4-55,31 \cdot \mathrm{t}_{\min }$ & 0,84 & 0,70 & 0,0000 \\
\hline $\mathrm{UF}_{\mathrm{Utah}}=872,3-62,73 \cdot \mathrm{t}_{\mathrm{md}}$ & 0,87 & 0,75 & 0,0000 \\
\hline \multicolumn{4}{|l|}{ Caçador } \\
\hline $\mathrm{HF}_{7,2}=735,1-126,5 \cdot \mathrm{t}_{\min }+5,654 \cdot \mathrm{t}_{\min }^{2}$ & 0,87 & 0,76 & 0,0015 \\
\hline $\mathrm{HF}_{7,2}=2041-258,3 \cdot \mathrm{t}_{\mathrm{md}}+8,272 \cdot \mathrm{t}_{\mathrm{md}}^{2}$ & 0,77 & 0,60 & 0,0161 \\
\hline $\mathrm{UF}_{\mathrm{Utah}}=-367,7+138,9 \cdot \mathrm{t}_{\min }-10,53 \cdot \mathrm{t}_{\min }^{2}$ & 0,73 & 0,53 & 0,0326 \\
\hline $\mathrm{UF}_{\mathrm{Utah}}=952,9-68,72 \cdot \mathrm{t}_{\mathrm{md}}$ & 0,96 & 0,93 & 0,0000 \\
\hline \multicolumn{4}{|l|}{ Bento Gonçalves } \\
\hline $\mathrm{HF}_{7,2}=1187-184 \cdot \mathrm{t}_{\min }+7,174 \cdot \mathrm{t}_{\min }^{2}$ & 0,92 & 0,84 & 0,0016 \\
\hline $\mathrm{HF}_{7,2}=2,359 \mathrm{E} 04-4768 \cdot \mathrm{t}_{\mathrm{md}}+321,1 \cdot \mathrm{t}_{\mathrm{md}}{ }^{2}-7,189 \cdot \mathrm{t}_{\mathrm{md}}{ }^{3}$ & 0,89 & 0,80 & 0,0158 \\
\hline $\mathrm{UF}_{\mathrm{Utah}}=534,9-50,5 \cdot \mathrm{t}_{\text {min }}$ & 0,81 & 0,66 & 0,0041 \\
\hline $\mathrm{UF}_{\mathrm{Utah}}=-2,741 \mathrm{E} 04+6132 \cdot \mathrm{t}_{\mathrm{md}}-448,4 \cdot \mathrm{t}_{\mathrm{md}}{ }^{2}+10,73 \cdot \mathrm{t}_{\mathrm{md}}{ }^{3}$ & 0,98 & 0,96 & 0,0001 \\
\hline \multicolumn{4}{|l|}{ Canela } \\
\hline $\mathrm{HF}_{7,2}=892,8-136 \cdot \mathrm{t}_{\min }+5,145 \mathrm{t}_{\min }^{2}$ & 0,93 & 0,86 & 0,0000 \\
\hline $\mathrm{HF}_{7,2}=1341-162,5 \cdot \mathrm{t}_{\mathrm{md}}+4,97 . \mathrm{t}_{\mathrm{md}}^{2}$ & 0,77 & 0,59 & 0,0019 \\
\hline $\mathrm{UF}_{\mathrm{Utah}}=404,7+10,26 \cdot \mathrm{t}_{\min }-4,55 \cdot \mathrm{t}_{\min }{ }^{2}$ & 0,88 & 0,77 & 0,0000 \\
\hline $\mathrm{UF}_{\text {Utah }}=1042-74,67 . \mathrm{t}_{\mathrm{md}}$ & 0,88 & 0,77 & 0,0000 \\
\hline
\end{tabular}

Rev. Ceres, Viçosa, v. 65, n.6, p. 474-480, nov/dez, 2018 
Nem sempre o modelo obtido com um elevado coeficiente de determinação (Tabela 4) manteve a eficiência nas estimativas, como em Caçador, que apresentou desempenho considerado 'péssimo' na estimativa mensal de horas-unidades de frio.

Em contrapartida, os coeficientes de determinação para Bom Jardim da Serra, Major Vieira e Bento Gonçalves mostram que as horas de frio calculadas conseguiram explicar entre 74 e $95 \%$ da variação das horas de frio mensal estimadas pelos modelos. Em relação ao EPE, as estimativas dos modelos dos referidos três municípios, apresentaram uma variação de 34 a 54,5 horas de frio por mês em relação às horas de frio calculadas (Tabela 5).

Os modelos obtidos para Major Vieira e Bento Gonçalves mantiveram o 'bom' e o 'ótimo' desempenhos, respectivamente, com os obtidos para a estimativa mensal de unidade de frio por Utah. Juntamente com Curitibanos que teve um 'ótimo' desempenho, as unidades de frio calculadas para esses três municípios explicaram entre 86 e $96 \%$ da variação das unidades de frio estimadas pelos modelos.

A variabilidade dos desempenhos na quantificação de frio entre os métodos e municípios é normal, numa região de estudo que apresenta diversidade de fatores climáticos (altitude, latitude, relevo, continentalidademaritimidade, entre outros). No estudo feito em todo o território acidentado do município de São Joaquim, na Serra catarinense, Iuchi et al. (2002) chamaram a atenção para a variabilidade observada nas condições térmicas e, consequentemente, para o acúmulo de horas-unidades de frio para macieiras jovens e adultas, mesmo sendo de um mesmo cultivar. Para algumas regiões do Paraná, Botelho et al. (2006) concluíram que os anos com invernos mais brandos apresentaram maiores diferenças entre o método de Utah e o das temperaturas horárias menores ou iguais a $7,2^{\circ} \mathrm{C}$. Cardoso et al. (2015) também comprovaram essa variabilidade do acúmulo de frio com a utilização de cinco métodos, durante nove anos, para a região de Vacaria, RS.

Considerando-se os modelos obtidos por municípios com melhor desempenho (c > 0,66), não houve uma unanimidade na tendência das estimativas de horas-unidades de frio de acordo com o erro médio. Contudo, chama a atenção que as estimativas feitas pelos modelos obtidos para Major Vieira foram as únicas a subestimar as horasunidades de frio, ocorrendo tendência oposta à dos demais municípios com desempenhos 'bom' ou 'ótimo'.

Da mesma forma que os resultados para Caçador e Bento Gonçalves tiveram, respectivamente, um 'péssimo' e um 'ótimo' desempenho nesta avaliação, é perfeitamente plausível que, em outros anos em que os modelos venham a ser avaliados, resultem em desempenhos diferentes. Tal avaliação fica como sugestão para trabalhos futuros, bem como avaliar os modelos para municípios próximos aos utilizados neste estudo e adotar outros métodos de quantificação de frio, como o de Utah Modificado, Carolina do Norte e Carolina do Norte Modificado, ou ajustá-los para dado local ou região.

Tabela 5: Avaliação da estimava mensal de horas de frio $\left(\mathrm{HF}_{7,2}\right)$, pelos modelos com maior coeficiente de determinação

\begin{tabular}{lccccc}
\hline $\mathbf{H F}_{7,2}$ & Curitibanos & Bom Jardim da Serra & Major Vieira & Bento Gonçalves & Caçador \\
\hline $\mathrm{EPE}$ & 47,21 & 33,99 & 54,50 & 39,15 & 64,31 \\
$\mathrm{EM}$ & $-26,29$ & 13,20 & $-29,84$ & 6,67 & 13,34 \\
$\mathrm{R}^{2}$ & 0,60 & 0,95 & 0,74 & 0,89 & 0,10 \\
$\mathrm{r}$ & 0,77 & 0,97 & 0,86 & 0,94 & 0,31 \\
$\mathrm{~d}$ & 0,83 & 0,97 & 0,85 & 0,95 & 0,62 \\
$\mathrm{c}$ & 0,64 & 0,94 & 0,73 & 0,89 & 0,19 \\
Desempenho & Mediano & Ótimo & Bom & Ótimo & Péssimo \\
\hline
\end{tabular}

$\overline{\mathrm{EPE}}=$ Erro padrão de estimativa; $\mathrm{EM}=$ Erro médio; $\mathrm{R}^{2}=$ coeficiente de determinação; $\mathrm{r}=$ coeficiente de correlação; $\mathrm{d}=$ Índice de concordância; $\mathrm{c}=$ Índice de confiança.

Tabela 6: Avaliação da estimativa de unidades de frio por Utah $\left(\mathrm{UF}_{\mathrm{Utah}}\right)$, pelos modelos com maior coeficiente de determinação

\begin{tabular}{|c|c|c|c|c|c|}
\hline $\mathbf{U F}_{\text {Utah }}$ & Curitibanos & Bom Jardim da Serra & Major Vieira & Bento Gonçalves & Caçador \\
\hline EPE & 38,35 & 101,05 & 152,60 & 37,10 & 107,84 \\
\hline EM & 23,93 & $-13,74$ & $-104,44$ & 15,84 & $-4,31$ \\
\hline $\mathrm{R}^{2}$ & 0,96 & 0,02 & 0,86 & 0,90 & 0,23 \\
\hline $\mathrm{r}$ & 0,98 & 0,14 & 0,93 & 0,97 & 0,48 \\
\hline $\mathrm{d}$ & 0,98 & 0,11 & 0,73 & 0,97 & 0,68 \\
\hline $\mathrm{c}$ & 0,96 & 0,02 & 0,68 & 0,94 & 0,33 \\
\hline Desempenho & Ótimo & Péssimo & Bom & Ótimo & Péssimo \\
\hline
\end{tabular}

$\overline{\mathrm{EPE}}=$ Erro padrão de estimativa; EM= Erro médio; $\mathrm{R}^{2}=$ coeficiente de determinação; $\mathrm{r}=$ coeficiente de correlação; $\mathrm{d}=$ Índice de concordância; $\mathrm{c}=$ Índice de confiança. 
Petri et al. (2006) ressaltam que os métodos de Utah e Carolina do Norte foram desenvolvidos para condições climáticas do Hemisfério Norte, em que os períodos de outono e inverno apresentam temperaturas com menores variações, enquanto nas condições do sul do Brasil ocorrem grandes oscilações térmicas no período hibernal. Já Botelho et al. (2006) mostraram que a quantificação de unidades de frio por Utah foi equivalente à obtida pelo método de Utah Modificado, sendo que essa diferença foi menor nas regiões paranaenses analisadas, em anos com invernos mais amenos.

\section{CONCLUSÕES}

Recomenda-se o uso da temperatura mínima e da média mensal do ar para a estimativa das horas de frio e das unidades de frio, respectivamente, para os locais estudados.

A oscilação climática do inverno no Rio Grande do Sul e em Santa Catarina resulta em desempenhos distintos dos modelos de estimativas de horas-unidades de frio.

\section{REFERÊNCIAS}

Botelho RV, Ayub RA \& Müller MML (2006) Somatória de horas de frio e unidade de frio em diferentes regiões do estado do Paraná. Scientia Agraria, 07:89-96.

Camargo AP \& Sentelhas PC (1997) Avaliação do desempenho de diferentes métodos de estimativa da evapotranspiração potencial no Estado de São Paulo. Revista Brasileira de Agrometeorologia, 05:89-97.

Cardoso LS, Bergamaschi H, Bosco LC, Paula VA de \& Nachtigal GR (2015) Unidades de frio para macieiras na região de VacariaRS, Brasil. Revista Brasileira de Fruticultura, 37:289-295.

Erez A (2000) Bud dormancy: Phenomenon, problems and solutions in the tropics and subtropics. In: Erez A (Ed.) Temperate fruit crops in warm climates. London: Kluwer Academic Publishers, p. 17-48.

Felippeto J (2008) Modelos de previsão da brotação para videira na serra gaúcha. Dissertação de Mestrado. Universidade Federal do Rio Grande do Sul, Porto Alegre. 126p.

Fioravanço JC \& Santos RSS dos (2013) Maçã: o produtor pergunta, a Embrapa responde. Brasília, Embrapa. 239p.

IBGE - Instituto Brasileiro de Geografia e Estatística (2018) SIDRA - Produção Agrícola Municipal: Maçã. Disponível em: https://sidra.ibge.gov.br/tabela/1613. Acessado em: 08 de novembro de 2018 .

Iuchi VL, Iuchi T, Brighenti E \& Ditrich R (2002) Quebra da dormência da macieira (Malus domestica Borkh) em São Joaquim-SC. Revista Brasileira de Fruticultura, 24:168-174.

Linvill DE (1990) Calculating chilling hours and chill units from daily maximum and minimum temperature observations. HortScience, 25:14-16.

Nachtigal GR, Fioravanço JC \& Hoffmann A (2009) Macieira. In: Monteiro JEBA (Org.) Agrometeorologia dos cultivos: o fator meteorológico na produção agrícola. Brasília, INMET. p.449-464.

Pereira AR, Angelocci LR \& Sentelhas PC (2002) Agrometeorologia Fundamentos e Aplicações Práticas. Guaíba, Livraria e Editora Agropecuária. 478p.
Petri JL, Palladini LA \& Pola AC (2006) Dormência e indução da brotação da macieira. In: EPAGRI (Ed.) A cultura da macieira. Florianópolis. p.261-298.

Pola AC, Bleicher J \& Bernardi J (1994) Avaliação de modelos de unidades e horas de frio para a previsão do início de brotação em macieira, cv. gala. Revista Brasileira de Fruticultura, 16:105118.

Rea R \& Eccel E (2006) Phenological models for blooming of apple in a mountainous region. International Journal of Biometeorology, 51:01-16.

Willmott CJ (1981) On the validation of models. Physical Geography, 02:184-194. 